\title{
Use of V-Agents and V-Analogue Compounds to Probe the Active Site of Atypical Butyrylcholinesterase from Oryzias latipes
}

Melanie G. Kirkpatrick ${ }^{\mathrm{a}}$, Robert C. diTargiani ${ }^{\mathrm{b}}$, Richard E. Sweeney ${ }^{\mathrm{c}}$, and Tamara C. Otto ${ }^{\mathrm{a}^{*}}$

Keywords: cholinesterase, nerve agent, organophosphorus, spontaneous reactivation

\begin{abstract}
The atypical butyrylcholinesterase (aBuChE) from Oryzias latipes shares approximately $65 \%$ sequence similarity to both acetylcholinesterase and butyrylcholinesterase and was studied for its capacity to spontaneously reactivate following inhibition by organophosphorus nerve agents. Like other cholinesterases, aBuChE was inhibited by all G- and V-type nerve agents. Interestingly, aBuChE was able to undergo spontaneous reactivation after inhibition with VR ( $\left.\mathrm{t}_{1 / 2}=5.5 \pm 0.2 \mathrm{~h}\right)$. Mass spectrometry of aBuChE after VR inhibition confirmed the presence of a covalently bound adduct of the size expected for non-aged VR on the peptide containing the active site serine. To understand the effect of substrate volume on rates of reactivation, the capacity of aBuChE to bind and spontaneously reactivate after inhibition with five $V$-agent analogues was examined. No appreciable reactivation was detected for enzyme
\end{abstract}


inhibited by V2 (VX with O-isopropyl on retained group), V4 (VX with $\mathrm{N}$-diethyl leaving group termination), or V5 (VX with $\mathrm{N}$-dimethyl leaving group termination). Minimal reactivation was detected with V1 (VX with O-propyl on retained group). Conversely, spontaneous reactivation was observed when aBuChE was inhibited by V3 (VX with O-isobutyl on retained group; $\mathrm{t}_{1 / 2}=$ $6.3 \pm 0.4 \mathrm{~h}$ ). The data suggest that the ability of aBuChE to spontaneously reactivate after inhibition by $\mathrm{V}$-agent analogues is related to the structure of the retained group. These results provide structural information that may shed light on the design of improved small molecule reactivators of nerve agent-inhibited acetylcholinesterase or butyrylcholinesterase, and further suggest that re-engineering the active site of a cholinesterase could result in enzymes with clinically relevant rates of nerve agent hydrolysis.

\section{Introduction}

Acetylcholinesterase (AChE) catalyzes the hydrolysis of the neurotransmitter acetylcholine (ACh) at the neuromuscular junction, thereby terminating neurotransmissions at the synapse. G- and V-type organophosphorus nerve agents are highly toxic compounds that covalently bind to and inhibit AChE at the active site serine ${ }^{1}$. This inhibition causes an excess of ACh at the synapse, resulting in a cholinergic crisis. Several drugs can be administered in a post-exposure scenario that either attempt to reactivate AChE (pralidoxime) or mitigate the toxic symptoms of nerve agent exposure (atropine, diazepam) $)^{2}$. However, these drugs do not prevent performance deficits, loss of consciousness, or potential brain damage. Research efforts have focused on the development of stoichiometric enzymes that can act as scavengers of nerve agents. Once administered, these bioscavengers would circulate through the bloodstream, bind to the nerve agents, and prevent them from reaching their physiological 
target. Butyrylcholinesterase (BuChE) is the leading stoichiometric scavenger of nerve agents for pre- ${ }^{3-6}$ and certain post-exposure situations ${ }^{7,8}$. Efforts have also concentrated on designing oximes that will reactivate agent-inhibited BuChE in circulation ${ }^{9,10}$.

The medaka Oryzias latipes (O. latipes) is a teleost fish with a vertebrate $\mathrm{AChE}^{11}$ and an atypical BuChE (aBuChE) $)^{12}$. The aBuChE is a cholinesterase with features intermediate to vertebrate $\mathrm{AChE}$ and $\mathrm{BuChE}{ }^{13-16}$. The aBuChE from O. latipes shares about $65 \%$ similarity between AChE and BuChE and can hydrolyze the substrates acetylthiocholine, butyrylthiocholine and propionylthiocholine. Although the origin of aBuChE from O. latipes is not known, this enzyme may represent an evolutionary intermediate cholinesterase between vertebrate $\mathrm{AChE}$ and $\mathrm{BuChE}^{12}$. The capacity of this novel cholinesterase to bind nerve agents has not been studied.

In this paper, we assessed whether aBuChE from O. latipes binds to G-and V-type nerve agents. We also examined whether agent-inhibited aBuChE can undergo spontaneous reactivation after nerve agent inhibition. Finally, $\mathrm{V}$-agent analogues were used to probe the fine specificity of the active site of the enzyme. Our results show that aBuChE may serve as an alternate platform for stoichiometric scavenger development. Structural information obtained from the use of $\mathrm{V}$-agent analogues with aBuChE may provide insight into the design of small molecules to improve the reactivation of nerve agent-inhibited AChE and BuChE.

\section{Materials and Methods}

2.1. Expression and purification of recombinant aBuChE. Trichoplusia ni (T. ni) larvae for either colony propagation or baculoviral infection were maintained as described ${ }^{17}$ by the staff at Chesapeake PERL, Inc. (Savage, MD). Larvae were infected with an orally active pre-occluded 
virus that encoded wild type aBuChE from O. latipes (NCBI reference sequence:

NP_001188442.1) with an N-terminal 6x-histidine tag. After clarification and filtration of homogenized insect larvae, recombinant aBuChE was affinity-purified using immobilized metalion affinity chromatography. Protein was eluted via an imidazole gradient. Purified recombinant aBuChE was kept at $-80^{\circ} \mathrm{C}$ and stored at $4{ }^{\circ} \mathrm{C}$ for short-term use.

2.2. SDS-PAGE and deglycosylation analysis. One-hundred micrograms of denatured recombinant aBuChE (Chesapeake PERL) was incubated with or without Protein Deglycosylation Mix (New England BioLabs, Ipswich, MA) for 4 hours at $37^{\circ} \mathrm{C}$, according to manufacturer's instructions. Protein ( $2 \mu \mathrm{g})$ was separated under denaturing conditions on a $10 \%$ miniPROTEAN ${ }^{\odot}$ TGX $^{\mathrm{TM}}$ precast gel (Bio-Rad, Hercules, CA). Protein was visualized using SimplyBlue ${ }^{\mathrm{TM}}$ SafeStain (Life Technologies, Grand Island, NY). Molecular weights were estimated by comparison to Precision Plus Protein ${ }^{\mathrm{TM}}$ Kaleidoscope ${ }^{\mathrm{TM}}$ Prestained Protein Standards (Bio-Rad).

2.3. Enzyme assays. Recombinant aBuChE ( $25 \mathrm{ng})$ was incubated in a 96 -well microplate with $2.5 \mu \mathrm{M}-4.5 \mathrm{mM}$ of either acetylthiocholine (AtCh), butyrylthiocholine (BtCh) or propionylthiocholine ( $\mathrm{PtCh}$ ) in the presence of two-fold excess 5,5'-dithiobis(2-nitrobenzoic acid) (DTNB) in $0.1 \mathrm{M} \mathrm{KPO}_{4}$ buffer, $\mathrm{pH} 7.4$. Turnover was followed at $A_{412}\left(\varepsilon=13,600 \mathrm{M}^{-1} \mathrm{~min}^{-1}\right)$ using a SpectraMax Plus 384 spectrophotometer (Molecular Devices, Sunnyvale, CA) at room temperature for $5 \mathrm{~min}$. Kinetic parameters were determined by Michaelis-Menten steady state kinetics using the equation $v=\mathrm{V}_{\max } *[\mathrm{~S}] /\left(K_{\mathrm{M}}+[\mathrm{S}]\right)$. Substrate inhibition was observed at high substrate concentrations of $\mathrm{BtCh}$ and PtCh, so those data were fit to $v=\mathrm{V}_{\max }{ }^{*}[\mathrm{~S}] /\left(K_{\mathrm{M}}+\right.$ $\left.[\mathrm{S}]^{*}\left(1+[\mathrm{S}] / K_{\mathrm{i}}\right)\right)$. The data from three independent experiments were fit to each model using 
Prism v5.04 (GraphPad, La Jolla, CA). $\mathrm{R}^{2}$ values for the nonlinear regression were greater than 0.99 .

2.4. Reactivation assays. Organophosphorus (OP) nerve agents were obtained from the U.S. Army Edgewood Chemical Biological Center (Aberdeen Proving Ground, MD). For reactivation assays, $4 \mu \mathrm{g}$ recombinant aBuChE was incubated with 20 -fold molar excess of racemic OP nerve agent for $10 \mathrm{~min}$ at room temperature. Uninhibited recombinant aBuChE was included as a positive control. After incubation, samples were passed through a Centri-Sep spin column (Princeton Separations, Adelphia, NJ) to remove unbound nerve agent. The eluate was diluted into $0.1 \mathrm{M} \mathrm{KPO}_{4}$ buffer, $\mathrm{pH} 7.4,1 \mathrm{mg} / \mathrm{mL}$ bovine serum albumin (BSA). The BSA was added to help stabilize aBuChE. At each time point, $50 \mu \mathrm{L}$ aliquots were removed and assayed for activity against $2 \mathrm{mM}$ PtCh, $4 \mathrm{mM}$ DTNB in $0.1 \mathrm{M} \mathrm{KPO}_{4}$ buffer, $\mathrm{pH} 7.4$, as described in Section 2.3. Measurements were taken over $23 \mathrm{~h}$ at room temperature using a Biomek NXP laboratory automation workstation (Beckman Coulter, Indianapolis, IN). Samples inhibited with nerve agent were compared to the uninhibited control, and results were plotted as a percentage of $100 \%$ total activity using one-phase association (Prism v5.04). Each experiment was conducted in duplicate, and the $\mathrm{R}^{2}$ values for the nonlinear regression were greater than 0.99 .

2.5. Trypsin digestion and mass spectrometry. Sixty micrograms of recombinant aBuChE was incubated with 20 -fold molar excess $\mathrm{V}$-agent for $10 \mathrm{~min}$ at room temperature. An uninhibited sample was included as a control. After incubation, samples were boiled for $10 \mathrm{~min}$ to denature the protein. A $1 \mu \mathrm{L}$ aliquot of $1 \mathrm{M} \mathrm{NH}_{4} \mathrm{HCO}_{3}$ was added to each tube to adjust the $\mathrm{pH}$ to 8.3 , and the samples were digested overnight at $37^{\circ} \mathrm{C}$ with $2.4 \mu \mathrm{g}$ Trypsin Gold, Mass 
Spectrometry Grade (Promega, Madison, WI). Trypsinized enzyme (10 $\mu \mathrm{L}$ ) was injected onto a BEH300 $\mathrm{C}_{18}$ column ( $2.1 \times 100 \mathrm{~mm}$; Waters, Milford, MA) on an Acquity UPLC system interfaced with a Synapt G2 high resolution mass spectrometer (Waters) to separate and analyze the peptides. The peptides were eluted with a 40 min gradient starting with $99 \%$ buffer $\mathrm{A}(0.1 \%$ formic acid in water) and ending with $40 \%$ buffer B ( $0.1 \%$ formic acid in acetonitrile) at a flow rate of $0.5 \mathrm{ml} / \mathrm{min}$. The mass spectrometer was operated in $\mathrm{MS}^{\mathrm{e}}$ and positive mode at $3.5 \mathrm{kV}$ and $120^{\circ} \mathrm{C}$.

\section{Results and Discussion}

3.1. Characterization of recombinant aBuChE expressed in T. ni larvae. Orally active baculovirus was used to direct the expression of recombinant aBuChE in T. ni larvae. The predicted molecular weight of recombinant aBuChE with a $6 \mathrm{x}$-histidine tag is approximately 63 kilodaltons $(\mathrm{kD})$. When the purified protein was analyzed by SDS-PAGE, three bands with the apparent molecular weights of 52, 66, and $68 \mathrm{kD}$ were observed (Figure 1). The 66 and $68 \mathrm{kD}$ bands migrated as a doublet and were difficult to resolve. To determine if the doublet was the result of glycosylation, the sample was incubated with a mix of five different deglycosylating enzymes. After separation via polyacrylamide gel electrophoresis, a mobility shift of the doublet was observed (Figure 1). The doublet resolved to an apparent molecular weight of 62 $k D$, which is similar to the predicted weight of the unmodified protein. This result suggests that the doublet represents alternative glycosylation of the core protein. Several novel bands appeared in the deglycosylated sample. These proteins represent several of the deglycosylation enzymes (O-glycosidase - 147 kD; $\beta$-N-acetylglucosaminidase - 71 kD; PNGase F - $36 \mathrm{kD}$ ). Tryptic digestion and mass spectrometry analysis of the $52 \mathrm{kD}$ band had about $40 \%$ 
coverage with aBuChE amino acid sequence; this band is most likely a degradation product (data not shown).

To assess the functional capacity of expressed aBuChE, the ability of the protein to hydrolyze acetylthiocholine (AtCh), butyrylthiocholine (BtCh), and propionylthiocholine (PtCh) was examined. Hydrolysis of each substrate was observed, and the kinetic constants are shown in Table 1. The enzyme binds more tightly to BtCh $\left(K_{M}=48 \pm 5 \mu \mathrm{M}\right)$ than to either AtCh or PtCh ( $K_{\mathrm{M}}=136 \pm 8 \mu \mathrm{M}$ and $92 \pm 7 \mu \mathrm{M}$, respectively). The recombinant enzyme has similar catalytic efficiencies $\left(k_{\text {cat }} / K_{\mathrm{M}}\right)$ for substrates AtCh and BtCh, while the $k_{\text {cat }} / K_{\mathrm{M}}$ for PtCh was about two-fold higher. These values are comparable to those reported by Pezzementi et al. $^{12}$ using recombinant aBuChE expressed in COS-7 monkey cells. Our results demonstrate that aBuChE is functionally active when expressed and purified from T. ni larvae.

\subsection{Examination of the capacity of recombinant aBuChE to bind OP nerve agents.}

Recombinant aBuChE was evaluated for its ability to bind G- and V-type nerve agents. The protein was incubated with 20 -fold molar excess of racemic nerve agent, and unbound nerve agent was removed by size-exclusion chromatography. To determine if the protein was inhibited by nerve agent, each sample was examined for its capacity to hydrolyze the substrate PtCh. None of the samples had detectable hydrolysis of PtCh when tested seven minutes after removal of excess nerve agent, indicating the enzyme was inhibited by both G-type and V-type nerve agents (Figure 2). The samples were tested again at 22 hours to assess levels of spontaneous reactivation. The enzyme had modest levels of spontaneous reactivation when inhibited by GB (7 $\pm 0.6 \%), G F(17 \pm 1 \%)$, and VX ( $8 \pm 3 \%)$. Interestingly, the enzyme regained 
complete activity $(100 \pm 9 \%)$ after inhibition with VR. No appreciable levels of reactivation were observed with GA or GD.

VX and VR are structural isomers. Because the enzyme fully reactivated after VR inhibition and not after inhibition with VX, we examined whether VR was adducted on the active site serine of recombinant aBuChE. Digestion of aBuChE with trypsin yields a 28 amino acid active site peptide VTLFGESAGAASVGFHLLSPASHGFFQR, with the active site serine (Ser203) shown in bold and underlined. The predicted monoisotopic mass for this peptide in the +3 and +4 charge states are 964.1599 and $723.3719 \mathrm{Da}$, respectively. These peaks should shift to 1008.8431 Da (+3) and 756.8843 Da (+4) when VR is adducted on Ser-203 and to 999.4994 Da $(+3)$ and 749.8765 Da (+4) when VX is adducted on Ser-203. The observed masses for each charge state and nerve agent were in close agreement to the expected values (Table 2). The results demonstrate that VR is covalently bound to the enzyme, and this bond must be broken for the enzyme to regain activity.

\subsection{Spontaneous reactivation of recombinant aBuChE inhibited with $V$-agent analogues.}

The recombinant aBuChE had different levels of spontaneous reactivation after inhibition with the structural isomers VX and VR. To investigate this finding, we examined the capacity of the enzyme to reactivate after inhibition by five $\mathrm{V}$-agent analogues. The analogues were designed such that the O-ethyl alkyl group of VX was replaced with O-propyl (V1), O-isopropyl (V2), or Oisobutyl (V3) on the retained group of the nerve agent. The N-diisopropyl alkyl groups of VX were replaced with $\mathrm{N}$-diethyl (V4) or $\mathrm{N}$-dimethyl (V5) on the leaving group (Figure 3). The V3 and V4 isomers are chimeras of VX and VR. V3 has the retained group of VR and the leaving group of VX, while V4 contains the retained group of VX and the leaving group of VR. After 
inhibition by VX, VR, or the V-agent analogues, spontaneous reactivation of recombinant aBuChE was followed at specific time intervals (Figure 4). The V3-inhibited enzyme had a comparable reactivation profile to that observed for VR. Conversely, enzyme inhibited with VX had no appreciable levels of reactivation. Analogues V2, V4, and V5 also had no noticeable levels of spontaneous reactivation. The data for these analogues were omitted from the figure for clarity. The reactivation profile for V1-inhibited recombinant aBuChE was about halfway between these two groups (Figure 4$)$. The reactivation half-time $\left(t_{1 / 2}\right)$ was determined for each data set (Table 3). As expected, VR and V3 had similar $t_{1 / 2}$ values $(5.5 \pm 0.2$ and $6.3 \pm 0.4 \mathrm{~h}$, respectively); these values were not statistically significant (t-test, $p=0.13$ ). The $t_{1 / 2}$ values for VX, V2, V4, and V5 could not be determined. Although enzyme inhibited with V1 showed appreciable reactivation, an accurate $t_{1 / 2}$ could not be established during the time period examined.

These results are in agreement with what is expected based on the structures of the Vagent analogues and their predicted adducts on Ser-203 of aBuChE. The predicted adduct on Ser-203 for V3 is identical to the VR adduct (Table 3), and the reactivation half-times for VR and V3 are comparable. Enzyme inhibited with VX, V4, or V5 would have the same adduct on Ser203; their reactivation profiles are similar. It was more difficult to predict if the enzyme would spontaneously reactivate when inhibited by V1 or V2, as these analogues have adducts that are different from both VX and VR. The spontaneous reactivation observed after inhibition by V1 supports the idea that an alkyl substituent with at least a three carbon extended chain is required for reactivation (Table 3). Because V2 had a similar reactivation profile to VX, V4, and 
V5, it appears that it is more difficult for aBuChE to undergo spontaneous reactivation with shorter carbon chains.

\section{Conclusions}

Here we show that recombinant aBuChE from O. latipes is inhibited by both $\mathrm{G}$ - and Vtype nerve agents. Additionally, recombinant aBuChE undergoes spontaneous reactivation after inhibition with VR, regaining full enzymatic activity. The enzyme also spontaneously reactivates after inhibition with GB, GF, and VX, but at much lower rates. Analogues of Vagents were used to probe the active site of recombinant aBuChE. By examining spontaneous reactivation rates with these analogues, it appears that the reactivation half-time is related to the functional group adducted on Ser-203. The data suggest that both the size and branched nature of the retained group affect the ability of the enzyme to spontaneously remove the adduct from the catalytic serine. Future studies will investigate the mechanism of spontaneous reactivation exhibited by aBuChE. Determining the reactivation half-times after inhibition with the stereoisomers of VR, V1, and V3 may shed light on the three-dimensional structure of the active site. Efforts to crystallize aBuChE are also in progress. Additional studies will be conducted with human AChE and BuChE to determine their behavior after inhibition with the $\mathrm{V}$ agent analogues. Finally, small molecule-mediated reactivation of agent-inhibited aBuChE will be explored. Together, these studies may provide a framework for improving the reactivation of human BuChE through modification of either a small molecule or the amino acid sequence of the enzyme. 


\section{References}

[1] Taylor, P. (2001) Anticholinesterase agents, In Goodman \& Gilman's The Pharmacological Basis of Therapeutics (Hardman, J. G., Limbird, L. E., and Gilman, A. G., Eds.), pp 175-192, McGraw-Hill, New York.

[2] Cannard, K. (2006) The acute treatment of nerve agent exposure, J Neurol Sci 249, 86-94.

[3] Broomfield, C. A., Maxwell, D. M., Solana, R. P., Castro, C. A., Finger, A. V., and Lenz, D. E. (1991) Protection by butyrylcholinesterase against organophosphorus poisoning in nonhuman primates, J Pharmacol Exp Ther 259, 633.

[4] Lenz, D. E., Maxwell, D. M., Koplovitz, I., Clark, C. R., Capacio, B. R., Cerasoli, D. M., Federko, J. M., Luo, C., Saxena, A., Doctor, B. P., and Olson, C. (2005) Protection against soman or VX poisoning by human butyrylcholinesterase in guinea pigs and cynomolgus monkeys, Chem Biol Interact 157-158, 205-210.

[5] Raveh, L., Grunwald, J., Marcus, D., Papier, Y., Cohen, E., and Ashani, Y. (1993) Human butyrylcholinesterase as a general prophylactic antidote for nerve agent toxicity. In vitro and in vivo quantitative characterization, Biochem Pharmacol 45, 2465.

[6] Saxena, A., Hastings, N. B., Sun, W., Dabisch, P. A., Hulet, S. W., Jakubowski, E. M., Mioduszewski, R. J., and Doctor, B. P. (2015) Prophylaxis with human serum butyrylcholinesterase protects Gottingen minipigs exposed to a lethal high-dose of sarin vapor, Chem Biol Interact 238, 161169.

[7] Mumford, H., Docx, C. J., Price, M. E., Green, A. C., Tattersall, J. E., and Armstrong, S. J. (2013) Human plasma-derived BuChE as a stoichiometric bioscavenger for treatment of nerve agent poisoning, Chem Biol Interact 203, 160-166.

[8] Mumford, H., M, E. P., Lenz, D. E., and Cerasoli, D. M. (2011) Post-exposure therapy with human butyrylcholinesterase following percutaneous VX challenge in guinea pigs, Clin Toxicol (Phila) 49, 287-297.

[9] Sit, R. K., Fokin, V. V., Amitai, G., Sharpless, K. B., Taylor, P., and Radic, Z. (2014) Imidazole aldoximes effective in assisting butyrylcholinesterase catalysis of organophosphate detoxification, $J$ Med Chem 57, 1378-1389.

[10] Radic, Z., Dale, T., Kovarik, Z., Berend, S., Garcia, E., Zhang, L., Amitai, G., Green, C., Radic, B., Duggan, B. M., Ajami, D., Rebek, J., and Taylor, P. (2013) Catalytic detoxification of nerve agent and pesticide organophosphates by butyrylcholinesterase assisted with non-pyridinium oximes, Biochem J 450, 231-242.

[11] Fluck, R. A. (1982) Localization of acetylcholinesterase activity in young embryos of the medaka Oryzias latipes, a teleost, Comp Biochem Physiol C 72, 59-64.

[12] Pezzementi, L., Nachon, F., and Chatonnet, A. (2011) Evolution of acetylcholinesterase and butyrylcholinesterase in the vertebrates: an atypical butyrylcholinesterase from the Medaka Oryzias latipes, PLoS One 6, e17396.

[13] Toutant, J. P., Massoulie, J., and Bon, S. (1985) Polymorphism of pseudocholinesterase in Torpedo marmorata tissues: comparative study of the catalytic and molecular properties of this enzyme with acetylcholinesterase, $J$ Neurochem 44, 580-592.

[14] Lundin, S. J. (1968) Properties of a cholinesterase from body muscles of plaice. (Pleuronectes platessa), Acta Chem Scand 22, 2183-2190.

[15] Stieger, S., Gentinetta, R., and Brodbeck, U. (1989) Cholinesterases from flounder muscle. Purification and characterization of glycosyl-phosphatidylinositol-anchored and collagen-tailed forms differing in substrate specificity, Eur J Biochem 181, 633-642.

[16] Leibel, W. S. (1988) Characterization of a pseudocholinesterase purified from surgeonfish tissues confirms the atypical nature of this enzyme, J Exp Zool 247, 198-208. 
[17] Liu, Y., DeCarolis, N., and Beek, N. (2007) Protein production with recombinant baculoviruses in lepidopteran larvae, Methods Mol Biol 388, 267-280.

\section{Acknowledgements}

This research was supported by the Defense Threat Reduction Agency - Joint Science Technology Office, Medical S\&T Division. Additionally, M.G.K. was supported in part by an appointment to the Postgraduate Research Participation Program at the U.S. Army Medical Research Institute of Chemical Defense administered by the Oak Ridge Institute for Science and Education through an interagency agreement between the U.S. Department of Energy and U.S. Army Medical Research and Materiel Command.

\section{Disclaimer}

The views expressed in this article are those of the authors and do not reflect the official policy of the Department of Army, Department of Defense, or the U.S. Government.

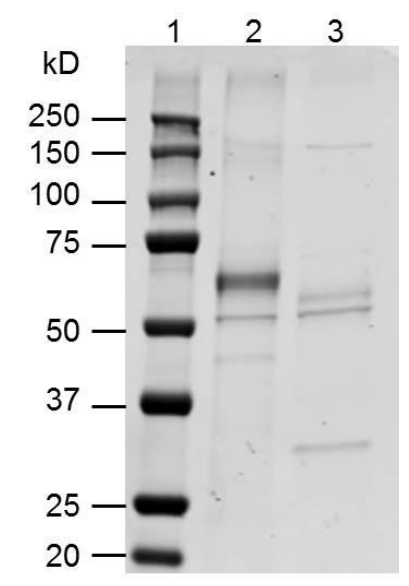

Figure 1. SDS-PAGE and deglycosylation analysis of purified recombinant aBuChE.

Recombinant aBuChE ( $2 \mu \mathrm{g})$ was separated under denaturing conditions and visualized with SimplyBlue ${ }^{\mathrm{TM}}$ SafeStain. Lanes are as follows: 1 . Molecular weight markers; 2 . Recombinant aBuChE; 3. Deglycosylated recombinant aBuChE. kD - kilodaltons. 
Table 1. Kinetic constants for recombinant aBuChE

\begin{tabular}{ccc}
\hline Substrate & $K_{\mathrm{M}}(\mu \mathrm{M})$ & $k_{\text {cat }} / K_{\mathrm{M}}\left(\mathrm{M}^{-1} \mathrm{~min}^{-1}\right)$ \\
\hline AtCh & $136 \pm 8$ & $1.7 \pm 0.41 \times 10^{8}$ \\
BtCh & $48 \pm 5$ & $1.3 \pm 0.13 \times 10^{8}$ \\
PtCh & $92 \pm 7$ & $3.1 \pm 0.85 \times 10^{8}$ \\
\hline
\end{tabular}

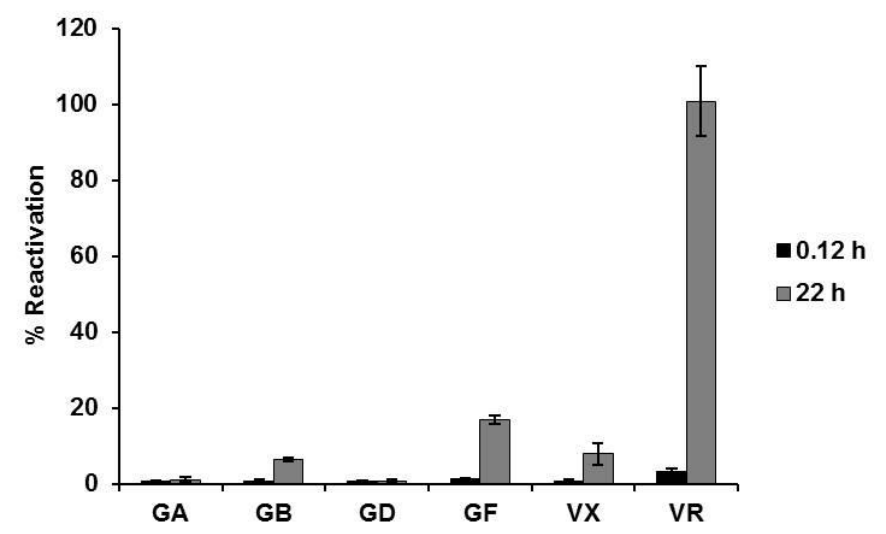

Figure 2. Percent spontaneous reactivation of nerve agent-inhibited recombinant aBuChE.

Recombinant aBuChE was incubated with a molar excess of tabun (GA), sarin (GB), soman (GD), cyclosarin (GF), VX, or VR $(n=3)$. The ability of the enzyme to hydrolyze PtCh was examined at $0.12 \mathrm{~h}$ (black bars) and $22 \mathrm{~h}$ (grey bars). The level of enzymatic activity is represented as a percent reactivation when compared to an uninhibited recombinant aBuChE control. Percent reactivation is shown \pm standard deviation.

Table 2. Expected and observed masses of tryptic peptide from $\mathrm{V}$-agent inhibited recombinant aBuChE ${ }^{1}$

\begin{tabular}{cccccc}
\hline Analyte & $\begin{array}{c}\text { Expected mass } \\
{[\mathrm{M}+3 \mathrm{H}]^{+3}}\end{array}$ & $\begin{array}{c}\text { Observed mass } \\
{[\mathrm{M}+3 \mathrm{H}]^{+3}}\end{array}$ & $\begin{array}{c}\text { Expected mass } \\
{[\mathrm{M}+4 \mathrm{H}]^{+4}}\end{array}$ & $\begin{array}{c}\text { Observed mass } \\
{[\mathrm{M}+4 \mathrm{H}]^{+4}}\end{array}$ & $\begin{array}{c}\text { Retention time } \\
(\mathrm{min})\end{array}$ \\
\hline aBuChE & 964.1599 & 964.1620 & 723.3719 & 723.3712 & 26.63 \\
aBuChE-VR & 1008.8431 & 1008.8523 & 756.8843 & 756.8822 & 28.18 \\
aBuChE-VX & 999.4994 & 999.4934 & 749.8765 & 749.8791 & 26.69 \\
\hline
\end{tabular}

${ }^{1}$ Each mass is expressed as daltons (Da). 
<smiles>CCOP(C)(=O)SCCN(C(C)C)C(C)C</smiles><smiles>[R]C(C)COP(C)(=O)SCCN(CC)CC</smiles><smiles>CCCOP(C)(=O)SCCN(C(C)C)C(C)C</smiles><smiles>CC(C)OP(C)(=O)SCCN(C(C)C)C(C)C</smiles><smiles>CCOP(C)(=O)SCCN(C)C</smiles><smiles>CC(C)COP(C)(=O)SCCN(C(C)C)C(C)C</smiles>

Figure 3. Structure of V-type nerve agents and V-agent analogues. 


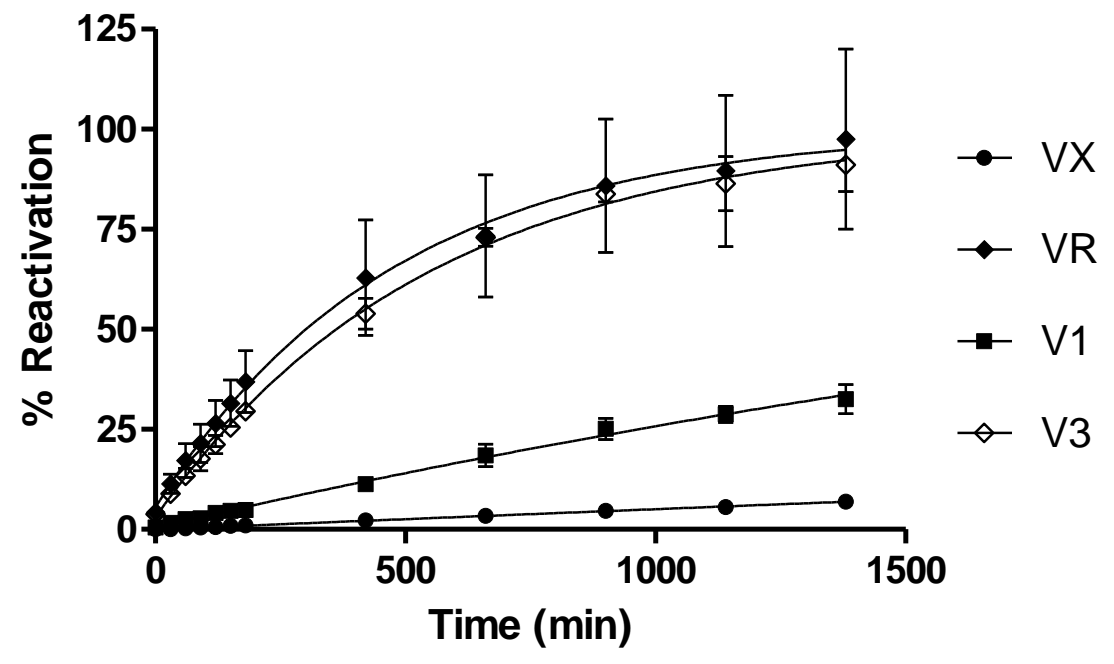

Figure 4. Percent reactivation of recombinant aBuChE after inhibition by V-type nerve agents

and V-agent analogues. Recombinant aBuChE was inhibited with a molar excess of each V-

agent and $V$-agent analogue $(n=2)$. At specific time points, the enzyme was examined for its capacity to hydrolyze PtCh. The level of enzymatic activity is represented as percent reactivation when compared to an uninhibited recombinant aBuChE control. Percent reactivation is shown \pm standard deviation. Reactivation profiles are shown for VX (solid circles), VR (solid diamonds), V1 (solid squares), and V3 (open diamonds).

\begin{tabular}{|c|c|c|c|c|c|c|c|}
\hline Agent & $\mathrm{VX}$ & V4 & V5 & VR & V3 & V1 & V2 \\
\hline$t_{1 / 2}(h)$ & N.D. & N.D. & N.D. & $5.5 \pm 0.2$ & $6.3 \pm 0.4$ & N.D. & N.D. \\
\hline $\begin{array}{c}\text { Predicted } \\
\text { adduct on } \\
\text { Ser-203 }\end{array}$ & $\mathrm{Se}$ & & & Ser' & & & $\mathrm{Ser}^{\mathrm{O}}$ \\
\hline
\end{tabular}

